\title{
Influencia de las creencias religiosas en las actitudes en el personal sanitario (P.S.) ante la muerte.
}

\author{
Influence of religious beliefs on the attitudes of health \\ professionals (H.P.) in the face of death.
}

R. Raja Hernández¹, F.J. Gala León², J.M. González Infante³,
M. Lupiani Giménez ${ }^{4}$, C. Guillén Gestososos, e I. Alba Sánchez ${ }^{6}$.

\section{RESUMEN}

Fundamento: Las creencias religiosas son una variable psicosocial de gran importancia para una proporción considerable de la población, entre la que se encuentran los Profesionales Sanitarios y, por consiguiente, influye en las actitudes que estos tienen hacia los demás. Por ello, es deseable investigar dichas influencias, sobre todo en situaciones críticas de la vida, entre las que se incluye la muerte por su trascedencia a la hora de la atención al enfermo terminal.

Objetivos y Metodología: Analizar las influencias de las creencias religiosas en las actitudes ante la muerte en el P.S. estableciendo su prevalencia y correlacionándolas con variables psicosociales y personalísticas, con otros aspectos religiosos, así como con la experiencia con moribundos; mediante técnicas psicométricas y un inventario de creencia religiosa, siguiendo un diseño observacionaltransversal, sobre una muestra representativa de la población del P.S. de un Hospital Universitario.

Resultados y Conclusiones:

-La mayoría del P.S. que componen la muestra resultan creyentes religiosos (con un nivel medio de práctica "moderado").

-Las mujeres se muestran más religiosas que los hombres y los jóvenes resultan menos religiosos que las personas mayores.

-De los dos grupos extremos bien diferenciados que aparecen (creyentes practicantes y no creyentes, por un lado y creyentes no practicantes, tibios en su fe, agnósticos, etc.,por otro) los que menos ansiedad ante la muerte manifiestan son los creyentes practicantes y no creyentes. -EI P.S., en general, presenta baja ansiedad (Estado y Rasgo) y baja ansiedad ante la muerte.

Palabras clave: actitudes, creencias religiosas, personal sanitario y creencias religiosas, creencias religiosas y muerte, actitudes hacia la muerte.

\section{ABSTRACT}

Basis: Religious beliefs are psychosocial variables of great importance for a large proportion of the population, including health professionals, and consequently influence the attitude which these people have towards others. It is therefore desirable to investigate said influences, above all in critical situations of life, among which death is included because of its transcendence in the case of the terminally ill.

Aims and Methodology: The analysis of the influence of religious beliefs on the attitudes of H.P. in the face of death, establishing their prevalence and correlating them with psychosocial and personality variables, with other religious aspects, as also with experience with the dying; by psychometric techniques and an inventory of religious beliefs, following an observational-transverse design, on a representative sample of the population of H.P. of a University Hospital.

Results and Conclusions:

-Most of the H.P. in the sample turned out to hold religious beliefs (with a moderate level of practise).

-Women were more religious than men, and the young less religious than the old.

-Of the two well defined groups which emerge (practising believers and non-believers on the one hand and nonpractising believers, those lukewarm in their faith, agnostics, etc., on the other) those who show least anxiety in the face of death are practising believers and non-believers.

-H.P. show less anxiety in general (State and Features) and low anxiety in the face of death.

Key words: attitudes, religious beliefs, health professionals and religious beliefs, religious beliefs and death, attitudes towards death.

1 Investigador, Doctor, del Grupo Investigador en Ps. de la Salud (PAI-CTS-386)

2 Catedrático de Ciencias Psicosociales Aplicadas. UCA.

3 Catedrático de Psiquiatría. UCA.

4 Profesora Titular de Enfermería Médica. UCA.

5 Profesor Titular de Psicología Social. UCA.

6 Investigador del Grupo de Investigación. 


\section{INTRODUCCIÓN:}

Investigar acerca de los aspectos psicológicos de las creencias religiosas (en los que se incluye el análisis de las actitudes que genera) es un campo de investigación de gran importancia si se parte de la concepción de que la religión, en mayor o menor grado y hoy por hoy, es una variable psicosocial importante en una proporción muy considerable de la población y que, por consiguiente, influye en las actitudes que esta población tenga hacia los demás.[I -4] Pero si esto es cierto para cualquier situación, más aún lo es en el caso de las situaciones críticas de la vida (entre las que está la muerte bien se trate de la de otros o la propia.) [5-12]

Así, en este trabajo nos hemos centrado en el análisis de las influencias de las creencias religiosas en las actitudes en el P.S. ante la muerte por las siguientes razones:

I. Porque el P.S. conforma un colectivo del que necesariamente vamos a tener que depender en algún momento de nuestra vida, de modo que nuestra salud o enfermedad van a quedar a merced de su pericia profesional y las actitudes que tengan hacia nosotros como pacientes, -sobre todo en las situaciones críticas y en los procesos terminales-.

2. Porque entre religión y muerte se establecen una serie de interacciones que van a influir, favorable o desfavorablemente, en las actitudes del P.S. (concretamente en la ansiedad y el temor ante la muerte que puedan experimentar) y condicionar su ejercicio profesional de forma positiva o negativa.

3. Porque aún hoy en los ambientes hospitalarios, donde las etapas finales de la vida y la aparición de la muerte son realidades cotidianas, se le otorga escaso valor a la influencia que tienen no solo las creencias religiosas del P.S. sino también a la ausencia de estas en las actitudes que van a desarrollar ante sus semejantes en el ejercicio de su profesión.

\section{OBJETIVOS:}

Con estas premisas hemos pretendido analizar la influencia de las creencias religiosas en las actitudes en el P.S. ante la muerte (medidas a través de la ansiedad manifestada ante ella), estableciendo la prevalencia de tales actitudes, correlacionándolas con variables psicosociales generales personalísticas, y otros aspectos religiosos, así como con la experiencia con moribundos, mediante técnicas psicométricas y un inventario de creencia religiosa.

Los objetivos que nos hemos planteado han sido:

- Establecer si las creencias religiosas influyen en las actitudes ante la muerte del P.S.

- Conocer la intensidad y la cualidad de tales actitudes .

- Analizar con qué variables psicosociales correlacionan estas actitudes.

- Identificar los grupos de especial atención en este campo.

\section{METODOLOGíA:}

Para cubrir tales objetivos hemos elegido un diseño observacional-transversal o de PREVALENCIA $[13,14]$ que se centra en un examen de una sección transversal de la población, examinando la relación entre los fenómenos estudiados [en este caso las actitudes -ansiedad- ante la muerte) y otras variables (entre ellas, las creencias religiosas) en un momento dado del tiempo, para obtener la prevalencia (la proporción de sujetos que en una población analizada y en un momento dado presentan las características estudiadas). 


\section{I. POBLACIÓN Y MUESTRA:}

Como población diana elegimos a los Médicos y Enfermeros del Hospital Universitario de Puerta del Mar de Cádiz, cifrados -según listado del P.S. que componía la plantilla en febrero de 1997- en I. 350 sujetos, con un $48.7 \%$ de varones y $51.2 \%$ de mujeres.

Sobre esta población $<<$ finita $>>$ (esto es, menor a 100.000 sujetos) [15] con un nivel de confianza de dos sigmas (955 por 1000) con un p menor al 0.005\% y un error estimado estándar de un 5\% máximo, el $\mathrm{N}$ de la muestra ha de ser de 300 elementos ( 154 mujeres y I 46 varones) según las tablas al uso del Harvard College [16]

Para elegirla, se efectuó un muestreo aleatorio simple, mediante la tabla de dígitos aleatorios de Fisher y Yates [17]. Atendiendo a que algunos no respondieran, otros fueran invalidados, etc., se eligieron 900 sujetos, con la proporción poblacional mujer/varón, es decir, el triple de la muestra precisa, con la esperanza de conseguir los elementos muestrales necesarios. Finalmente se consiguieron 333 elementos válidos adecuadamente estratificados por sexo: 187 mujeres (56.2 \%) y 146 varones (43.8\%).

\subsection{INSTRUMENTOS Y MATERIAL:}

El material e instrumentos utilizados han sido:

- Una hoja de presentación, en la que se asegura la confidencialidad, se dan instrucciones generales y se motiva a contestar.

- Un cuestionario sociodemográfico donde se han incluido: unos datos sociodemográficos de carácter general, una pregunta relacionada con la muerte, dos preguntas relacionadas con pacientes terminales y tres en torno a las creencias religiosas.

- El cuestionario STAI (A/R) (A/E) de Spielberger [I 8], en su adaptación española de Nicolás Seisdedos, que evalúa dos tipos de ansiedad: ansiedad "estado" (A/E) y "rasgo" (A/R).

- La Escala de Ansiedad ante la Muerte "DAS-I" de Templer [19], que mide cuatro factores comunes a este tipo de ansiedad: el cognitivo, físico, noción del tiempo y miedo a una muerte dolorosa.

- Un Inventario de Creencia Religiosa, de elaboración propia [20], que mide el nivel de creencia religiosa, no en términos teológicos sino sociológicos, independientemente del tipo de que se trate, como valor que la persona puede tener incorporado a su personalidad.

\subsection{RECOGIDA DE DATOS Y TRATAMIENTO:}

A cada sujeto que formaba parte de la muestra se le entregó un sobre en el que se le incluía otro para que el sujeto nos remitiera, de forma anónima y según las instrucciones que se incluían, la batería de pruebas una vez cumplimentada.

La recogida de datos se efectuó de marzo a septiembre de 1999, obteniendo de cada sujeto: una serie de datos psicosociales, información acerca de si ha tenido experiencias personales en relación con la muerte, si ha trabajado o no con pacientes terminales, consideración subjetiva de ser o no creyente religioso, consideración subjetiva sobre el grado en que practican o no sus creencias religiosas y una puntuación en la Escala DAS- I, en el Inventario de Creencia Religiosa y las Escalas S y R (del STAI).

Tales datos fueron procesados mediante el programa informático $<<$ EPI-INFO $>>$ del C.D.C. /Atlanta) en su versión 6.04 [21,22], obteniéndose las puntuaciones directas y porcentuales en cada opción de cada ítem del Cuestionario Sociodemográfico, en toda la muestra; obteniéndo- 
se lo mismo en las subescalas S y E del STAI, DAS- I y el Inventario de Creencia Religiosa. Asimismo calculamos las pruebas de significación (Chi cuadrado) para los datos no porcentuales.

\section{RESULTADOS Y DISCUSIÓN:}

\section{I. Generales. (Ver tabla I)}

a) Aparecen más mujeres que hombres, lo cual es una tónica general en las poblaciones sanitarias, ya que estos mismos datos también aparecen en otros trabajos anteriores. [23] Estas son más jóvenes y son mayoría en el grupo de los solteros, lo que obviamente está vinculado a la edad. Por otra parte hay más mujeres que han tenido experiencias personales con la muerte, aunque hay menos que han trabajado con pacientes terminales que los hombres.

b) La edad más representativa se establece entre los $31-40$ años, siendo mayor en las categorías que requieren especialización (doctores y médicos especialistas) y dándose un mayor porcentaje de personas de mayor edad que han tenido experiencias personales con la muerte, mientras que hay un mayor porcentaje de menor edad que no las han tenido.

En cuanto al criterio de considerarse o no creyente religioso, aún siendo una muestra y una población que se manifiesta eminentemente creyente, el mayor porcentaje de los que se consideran "no creyentes" se da entre los menores de 40 años, encontrándose la inmensa mayoría de los que sí se consideran "creyentes" a partir de los $4 \mathrm{I}$ años de edad. Además, a medida que aumenta la edad, la consideración del nivel en que practican sus creencias los sujetos de la muestra es mayor. Estos resultados coinciden con la opinión popular de que con la edad la persona se hace más religiosa. Lo que no tiene porqué suponer mayor grado de creencia dogmática y una práctica acorde.[24]

c) El grupo de los casados es mucho más numeroso que el de los solteros. Y la mayor proporción de estos últimos se ubica en la categoría de médicos no especialistas, mientras que la de los casados es mayor en la categoría de doctor.

Por otra parte, las experiencias personales con la muerte son más propias de los casados que de los solteros, por la edad. Y en cuanto a considerarse o no creyente religioso, no se han encontrado diferencias significativas entre casados y solteros (aunque en el grupo de los solteros se aprecia un ligero aumento de los no creyentes, por la edad)

d) En cuanto a la categoría profesional, los médicos no especialistas muestran un mayor porcentaje en no haber tenido experiencias personales con la muerte. $Y$ no se han encontrado diferencias significativas entre esta variable y "considerarse o no creyente religioso" ni con el "nivel de práctica religiosa"

e) Del análisis de los resultados en la variable "área hospitalaria" destaca que el área más representativa es el área médica, aunque es en el área quirúrgica donde se encuentran los que se consideran más practicantes de sus creencias religiosas.

f) Igualmente, lo más relevante en cuanto a la "antigüedad profesional" es que en el grupo de los sujetos con una antigüedad profesional entre los I I-20 años es en el que está el mayor porcentaje de los que se consideran creyentes religiosos. 
g) No se han encontrado diferencias significativas entre "haber trabajado o no con pacientes terminales" y el "considerarse o no creyente religioso" ni con el "nivel en que los sujetos consideran que practican sus creencias religiosas", aunque sí se aprecia una pequeña diferencia en el grupo de los que han trabajado con pacientes terminales puesto que en este grupo tiene una mayor representatividad los que se muestran no creyentes. Pensamos que en la base de estos resultados podría estar influyendo una actitud de resentimiento contra la religión y sus figuras simbólicas con motivo de experimentar la muerte como algo "sin sentido" -como un fracaso- [25] que viene a truncar las motivaciones básicas de la vida (ilusiones, proyectos, expectativas, disfrute de bienes materiales, familiares, etc.) - sobre todo cuando aún se es joven- produciéndose así un alejamiento de todo lo relacionado con lo religioso.

h) En cuanto al criterio particular de considerarse o no creyente religioso, resulta que una proporción muy considerable de la muestra (casi el $85 \%$ ) se considera creyente; con lo que tenemos que el P.S. de la muestra se considera creyente por encima de la media española $(71,5 \%)$ y la europea (67\%) [26].

i) Frente a lo anterior tenemos que solo un $47,6 \%$ de la muestra considera que el nivel en que practican sus creencias religiosas es moderado. Lo que no deja de llamarnos la atención. La explicación de estos resultados estaría en la diferencia que existe entre "ser religioso" -tener unas costumbres religiosas- $y$ "ser creyente religioso" -tener unas creencias religiosas que se practican como forma de vida-[27].

Por otra parte no aparecen diferencias significativas en cuanto a esta variable en relación al sexo, sin embargo, aunque las mujeres se consideran porcentualmente más creyentes que los hombres, esto no se traduce en una mayor percepción de práctica religiosa.

En cuanto a la edad, se observa que en el grupo que se considera practicante las edades son más avanzadas que en el que se considera no practicante. La explicación de estos resultados va en la línea de lo referido anteriormente: la practica religiosa y no solo la autoconsideración de ser creyente -como en el caso de las mujeres- correlaciona con una mayor edad, como también lo refrenda la literatura científica al respecto.[28].

\subsection{InVENTARIO de CReENCIA Religiosa. (Ver Tabla 2 y Gráfico I)}

A diferencia de los resultados expuestos obtenidos del criterio subjetivo que los sujetos de la muestra tienen, vamos a exponer a continuación los obtenidos con el Inventario de Creencia Religiosa que intenta medir de forma "objetiva" la creencia religiosa y su práctica, viendo la correlación que se establece entre ellos.

a) Como podemos apreciar, la mayoría del P.S. que componen la muestra también resultan de forma objetiva creyentes religiosos (y con un nivel medio de práctica "moderado"), las mujeres se muestran más religiosas que los hombres, los menores de 30 años conforman el grupo menos religioso (lo que concuerda con la opinión popular de que con la edad la persona se vuelve más religiosa. Esto se debe a que -como indica la psicología evolutiva $[29,30]$ - muchos valores y actitudes sobre los que se constituye la personalidad, y con ellos las "creencias religiosas", adquieren su valor e importancia para las personas a partir de una edad en la cual se ha consolidado una determinada madurez) 
b) En lo referente a considerarse o no creyente religioso, el grupo que supuestamente no se considera creyente religioso presenta los mayores porcentajes en los niveles más bajos del Inventario de Creencia Religiosa, e incluso no tiene representación en los niveles más elevados (es decir, -como era de esperar- no puntúan en esta prueba "objetiva" como creyentes).

c) Igualmente, en cuanto a la práctica de las creencias religiosas, los que se consideran "no practicantes", presentan los mayores porcentajes en los niveles más bajos del inventario, mientras que, los que se consideran "plenos practicantes" presentan mayores porcentajes en los niveles más elevados del inventario.

\section{GRÁFICO 1: DISTRIBUCIÓN DE LA MUESTRA SEGÚN LOS RESULTADOS GLOBALES EN EL INVENTARIO DE CREENCIA RELIGIOSA.}

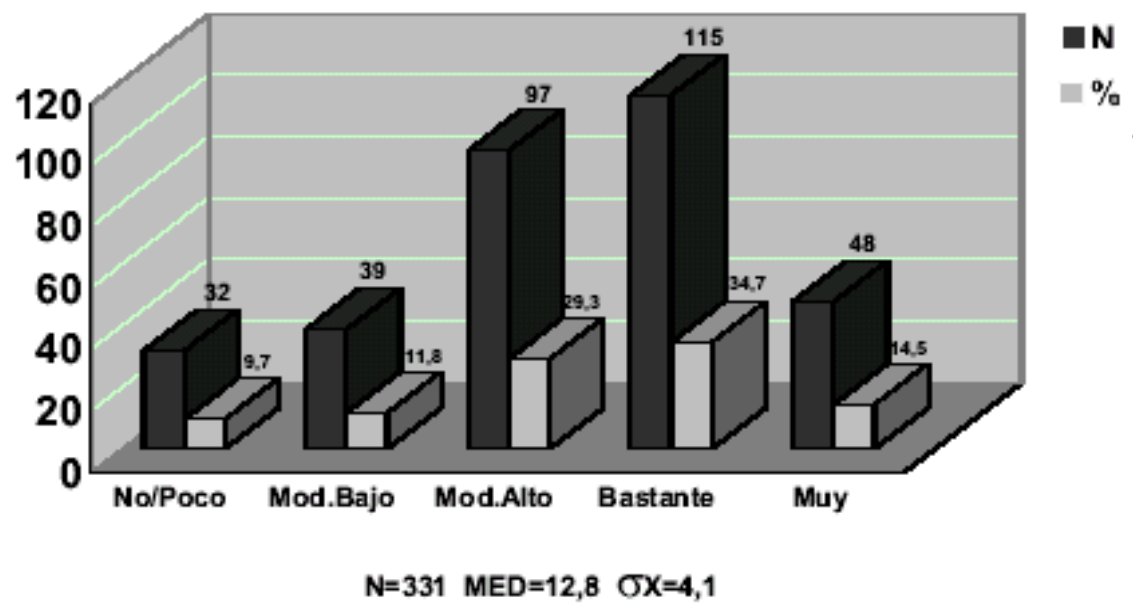

\subsection{Escala DAS-I (ANSIEDAd ANTE LA MUERTE) (Ver Tabla 3 y Gráfico 2)}

a) La ansiedad media ante la muerte del P.S. de la muestra es moderada baja. Lo cual es un dato importante a favor de este colectivo, dado lo saludable de esta actitud en el ejercicio profesional. [3|-33].

b) En cuanto a la edad, los niveles más elevados de ansiedad ante la muerte se dan mayoritariamente entre los 4I-50 años (ningún menor de 30 años presenta "mucha" ansiedad).

Estos datos siguen la tónica general del sentido común que considera que conforme se acerca uno al final de la vida, aumenta la ansiedad que se tiene ante la muerte, pero no se muestran coincidentes con los resultados obtenidos en los trabajos [34-36] que no encuentran relación significativa entre edad y ansiedad ante la muerte y que justifican esta ausencia de ansiedad por la intervención de mecanismos de defensa tales como, la negación y represión; quizás estos mecanismos de defensa no actúan del mismo modo en el P.S. que tiene más presente de forma cotidiana la experiencia de la muerte, y por ende, le es más difícil establecerlos, tal y como sucede en otras poblaciones analizadas. [37] 
Por otra parte, tan solo un 13\% de los sujetos a estas edades presenta niveles bajos (poca o ninguna ansiedad ante la muerte). Todo ello concuerda perfectamente con lo que indica la psicología del desarrollo [38,39] más actual: en cuanto a que las personas más mayores tienden a pensar en la muerte más frecuentemente que los jóvenes oscilando las reacciones entre dos polos: serenidad y angustia-ansiedad.

c) La antigüedad profesional que más se ve afectada por esta ansiedad es la de más de 20 años (lo cual correlaciona con la edad).

d) Y es en el área quirúrgica donde mayor porcentaje se registra en el nivel de "mucha" ansiedad ante la muerte (tanto por el mayor contacto con la experiencia de la muerte "directa" como por tener los niveles medios de edad mayores).

e) También los mayores niveles de ansiedad ante la muerte se dan en sujetos con más ansiedad "rasgo". Lo que es lógico dada la correlación que existe entre este tipo de ansiedad y la ansiedad en general.

f) En cuanto a la creencias religiosas y sus prácticas, aunque no se constatan grandes diferencias -téngase en cuenta que la muestra es eminentemente creyente y esto dificulta la discriminación de las diferencias- sí vemos como los practicantes religiosos son los que manifiestan menos ansiedad ante la muerte.

Con relación a estos resultados, unos estudios [40,4I] vienen a indicar la existencia de relaciones significativas entre religión (amortiguadora) y ansiedad ante la muerte; y otros $[42,43]$ que esta relación no existe o es insignificante. Esto nos hace considerar lo que Templer y Dotson ( 1970) [44] indican acerca de la posibilidad de que la religión no constituya un valor considerable en la vida de muchas personas, con lo cual no sería un determinante importante del nivel de ansiedad ante la muerte (salvo que esta religiosidad se traduzca en práctica real o forma de vida).

\section{GRÁFICO 2: DISTRIBUCIÓN DE LA MUESTRA SEGÚN LOS RESULTADOS GLOBALES EN LA ESCALA DAS-1 (ANSIEDAD ANTE LA MUERTE).}



$\mathrm{N}=333 \quad \mathrm{MED}=6,7 \quad \sigma \mathrm{X}=2,0$ 
Por otra parte, esta ambigüedad también podría justificarse sobre la base de la dificultad que surge a la hora de operativizar la "religión" por varios motivos; tales como:

- La cantidad de religiones diferentes que existen.

- La cantidad de "grupos religiosos" diferentes que pertenecen incluso a una misma religión.

- Pero, sobre todo, por las diferencias entre "religión meramente formalista" o de pertenencia y "religión traducida en forma de vida y en práctica" (recordemos que nosotros medimos ambas).

Curiosamente, y esto es un dato muy importante, sí aparecen diferencias entre los subgrupos extremos (creyentes muy practicantes y los no creyentes) y el resto pudiendo ser considerados como un subgrupo respecto a los intermedios (creyentes no practicantes, etc.) de modo que quienes menos ansiedad manifiestan son los subgrupos extremos (creyentes muy practicantes y los no creyentes). Así pues, nos aparecen como amortiguadores ante este tipo de ansiedad tanto el "ateísmo puro" como las "fuertes y coherentes creencias religiosas" y es como si ambas certezas aliviaran el temor ante la muerte, quedando más indefensos los intermedios, agnósticos o tibios en su fe, en la línea de lo que comentan Ramos y García. [45].

\subsection{EsCALA STAI - R/E (ANSIEDAD RASGO Y ESTADO) (Ver Tablas 4 y 5 y Gráficos 3 y 4)}

a) La media en A/R del P.S. de la muestra es moderada baja (lo cual, como en el caso de la ansiedad ante la muerte, es deseable en el ejercicio profesional) $[46,47]$

b) Los hombres ofrecen más $\mathrm{A} / \mathrm{R}$ que las mujeres profesionales. Estos resultados parecen contradecir la opinión popular de que las mujeres son más ansiosas que los hombres y no están en consonancia con los trabajos [48] que indican que las mujeres suelen obtener puntuaciones más elevadas en ansiedad que los hombres, tanto en A/R como A/E. Sin embargo, esta aparente contradicción se salva si consideramos que estamos hablando de una muestra de mujeres muy particular y como tal muy sesgada respecto al total del colectivo femenino -en nuestro caso todas las mujeres tienen estudios superiores-, y en este caso, como nos indica la psicología diferencial, la diferencia con los hombres en rasgos y actitudes se minimizan concordando más por estatus y profesión que por sexo. [49,50]

c) A medida que aumenta la edad aumenta la A/R.

d) La categoría que manifiesta mayor A/R es la de "médico no especialista".

Este aumento de ansiedad en esta categoría profesional, tendría su explicación en [5 I-53] que parte de estos médicos no especialistas son principiantes y su experiencia profesional es escasa, por lo que presentan más ansiedad que los que tienen más experiencia en la profesión.

e) Se da una correspondencia equivalente entre la $A / R$ y la $A / E$ ( lógico, dada la relación que existe entre ambas) Así pues, la A/E media de la muestra también es moderada baja, y la categoría profesional que mayor A/E presenta es la de "médico no especialista". 
GRÁFICO 3: DISTRIBUCIÓN DE LA MUESTRA SEGÚN LOS RESULTADOS GLOBALES EN LA ESCALA STAI-R (ANSIEDAD RASGO).

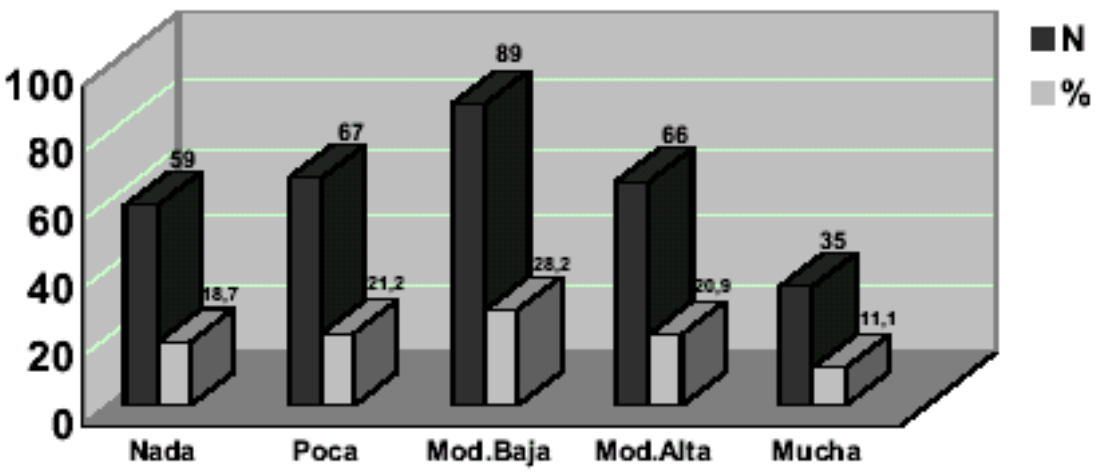

$\mathrm{N}=316 \quad \mathrm{MED}=35,7 \quad \sigma \mathrm{X}=27,4$

GRÁFICO 4: DISTRIBUCIÓN DE LA MUESTRA SEGÚN LOS RESULTADOS GLOBALES EN LA ESCALA STAI-E (ANSIEDAD ESTADO).

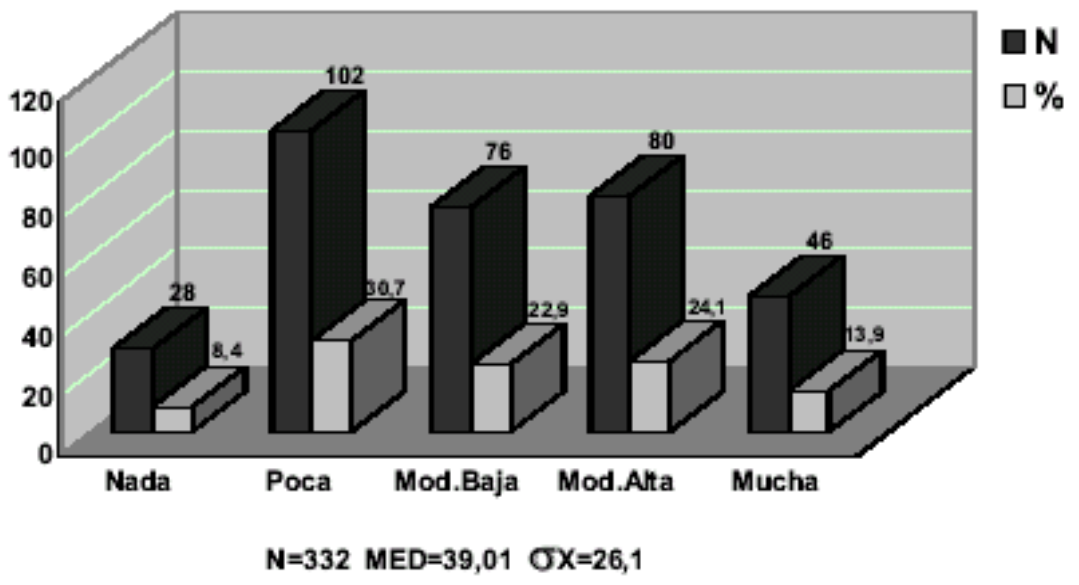

\section{CONCLUSIONES:}

A la vista de lo dicho podemos concluir que:

- En relación a la RELIGIOSIDAD.

I. Se constata, en el P.S., la tendencia a considerarse como "creyente religioso", por el mero hecho de pertenecer a alguna religión lo que no se traduce necesariamente en prácticas religiosas acordes. Aunque también es importante el grupo que discrimina entre tener una creencia religiosa que se vivencia y practica y la mera "religiosidad nominativa".

2. La mujer resulta más religiosa y practicante de sus creencias que el hombre. 
3. El haber tenido o no experiencias personales con la muerte de personas próximas no se muestra como un factor influyente en las creencias y prácticas religiosas del personal sanitario.

4. El haber trabajado con pacientes terminales sí parece influir de forma negativa en la dimensión religiosa del personal sanitario, produciéndose un cierto alejamiento con todo lo relacionado con la religión.

5. La correlación positiva encontrada entre el criterio subjetivo de considerarse "creyente" y "practicante religioso" y los resultados obtenidos en el Inventario de Creencia Religiosa que hemos construido y utilizado para este trabajo, pone de manifiesto su validez y fiabilidad.

6. Con la edad, el P.S. no solo experimenta un acercamiento a la actitud religiosa de tipo social sino que, además, aumenta el grado de interiorización o creencia del dogma religioso que profesa.

\section{- En relación con la ANSIEDAD.}

I. No se aprecian diferencias significativas entre los hombres y las mujeres P.S. en cuanto a la ansiedad ante la muerte.

2. El haber tenido experiencias personales con la muerte sí se muestra como un factor influyente aumentando el nivel de ansiedad que se siente ante la muerte, pero no en cuanto a la ansiedad (R/E)

3. El haber trabajado o no con pacientes terminales no parece influir en el P.S.en cuanto a la ansiedad experimentada ante la muerte, ni en la ansiedad (R/E) que se experimenta.

4. Tanto el considerarse "muy creyente religioso" como "no creyente religioso" no parece tener ninguna influencia sobre la ansiedad ante la muerte que se siente. Lo que sí se muestra influyente es ser o no practicante de estas creencias. Así se ha puesto de manifiesto que en los subgrupos extremos (los muy creyentes-practicantes y en los absolutamente no creyentes) la ansiedad ante la muerte es menor que en los subgrupos intermedios (creyentes no practicantes, religiosos nominativos, etc.)

5. Los índices de ansiedad "rasgo" y "estado" que presenta el P.S. en general son bajos.

6. Los factores de tipo social como el nivel de estudios, estatus y profesión, que minimizan las diferencias entre hombres y mujeres en actitudes, también minimizan las diferencias en A/R y A/E que se da en el P.S. por razón de sexo.

7. El estar casado o en situación similar es un factor social que se muestra influyente en la A/R y A/E amortiguándolas. Sin embargo, la condición de estar o no casado no parece tener influencia en la ansiedad ante la muerte.

8. La escasa experiencia profesional del P.S., independientemente que pertenezca a la clase médica o de enfermería, influye aumentando la ansiedad (R y E) que sufre. 
Tabla 1.- RESULTADOS GLOBALES EN LA MUESTRA TOTAL

Hospital Universitario "PUERTA DEL MAR" Cádiz

\begin{tabular}{|c|c|c|c|c|}
\hline & $\mathbf{N}$ & $\%$ & TOTAL & NO CONT. \\
\hline TODOS & 333 & 100 & 333 & \\
\hline VARÓN & 146 & 43.8 & & \\
\hline MUJER & 187 & 56.2 & 333 & \\
\hline$<30$ AÑOS & 24 & 7.2 & \multirow{4}{*}{332} & \multirow{4}{*}{1} \\
\hline $31-40$ ANOS & 162 & 48.8 & & \\
\hline 41-50 ANOS & 123 & 37.0 & & \\
\hline$>50$ ANOS & 23 & 6.9 & & \\
\hline$\overline{\text { SOLTERO }}$ & 61 & 19.9 & \multirow{2}{*}{307} & \multirow{2}{*}{26} \\
\hline CASADO & 246 & 80.1 & & \\
\hline DOCTOR & 42 & 12.6 & \multirow{5}{*}{333} & \\
\hline MÉDICO NO ESPECIALISTA & 36 & 10.8 & & \\
\hline MEDICO ESPECIALISTA & 33 & 9.9 & & \\
\hline D.U.E. & 182 & 54.7 & & \\
\hline A.T.S. & 40 & 12.0 & & \\
\hline ÁREA MÉDICA & 82 & 30.7 & \multirow{3}{*}{267} & \multirow{3}{*}{66} \\
\hline AREA QUIRÜRGICA & 74 & 27.7 & & \\
\hline OTRAS AREAS & 111 & 41.6 & & \\
\hline HASTA 10 AÑOS & 88 & 26.7 & \multirow{3}{*}{330} & \multirow{3}{*}{3} \\
\hline 11-20 ANOS & 155 & 47.0 & & \\
\hline MÁS DE 20 ANOS & 87 & 26.4 & & \\
\hline SI, FAMILIARES & 166 & 49.8 & \multirow{3}{*}{333} & \\
\hline SI, AMIGOS & 144 & 43.2 & & \\
\hline NO & 23 & 6.9 & & \\
\hline SI TRABAJA CON P. TERMINAL & 263 & 79.2 & \multirow{2}{*}{332} & \multirow{2}{*}{1} \\
\hline NO TRABAJA CON P. TERMINAL & 69 & 20.8 & & \\
\hline SI ES CREYENTE RELIGIOSO & 279 & 84.5 & \multirow{2}{*}{330} & \multirow{2}{*}{3} \\
\hline NO ES CREYENTE RELIGIOSO & 51 & 15.5 & & \\
\hline NO PRACTICANTE & 49 & 16.9 & \multirow{3}{*}{290} & \multirow{3}{*}{43} \\
\hline PRACTICANTE MODERADO & 138 & 47.6 & & \\
\hline SI PRACTICANTE & 103 & 35.5 & & \\
\hline STAI R & $\begin{array}{c}x \\
35.7\end{array}$ & $\begin{array}{c}\sigma x \\
27.4\end{array}$ & 316 & 17 \\
\hline STAI E & $\begin{array}{c}x \\
39.1\end{array}$ & $\begin{array}{c}\sigma x \\
26.1\end{array}$ & 332 & 1 \\
\hline DAS 1 & $\begin{array}{c}x \\
6.7\end{array}$ & $\begin{array}{c}\sigma x \\
2.0\end{array}$ & 333 & \\
\hline INVENT. CREENCIA RELIG. & $\begin{array}{c}x \\
12.8\end{array}$ & $\begin{array}{l}\sigma x \\
4.1\end{array}$ & 331 & 2 \\
\hline NADA ANSIEDAD RASGO & 59 & 18.7 & \multirow{5}{*}{316} & \multirow{5}{*}{17} \\
\hline POCA ANSIEDAD RASGO & 67 & 21.2 & & \\
\hline MODERADA BAJA ANS. RASGO & 89 & 28.2 & & \\
\hline MODERADA ALTA ANS. RASGO & 66 & 20.9 & & \\
\hline MUCHA ANSIEDAD RASGO & 35 & 11.1 & & \\
\hline NADA ANSIEDAD ESTADO & 28 & 8.4 & \multirow{5}{*}{332} & \multirow{5}{*}{1} \\
\hline POCA ANSIEDAD ESTADO & 102 & 30.7 & & \\
\hline MODERADA BAJA ANS. ESTADO & 76 & 22.9 & & \\
\hline MODERADA ALTA ANS. ESTADO & 80 & 24.1 & & \\
\hline MUCHA ANSIEDAD ESTADO & 46 & 13.9 & & \\
\hline NADA/POCA ANS. ANTE MUERTE & 49 & 14.7 & & \\
\hline MODERADA BAJA ANS. MUERTE & 172 & 51.7 & & \\
\hline MODERADA ALTA ANS. MUERTE & 100 & 30.0 & 333 & \\
\hline MUCHA ANSIEDAD A LA MUERTE & 12 & 3.6 & & \\
\hline NO/POCO CREYENTE RELIGIOSO & 32 & 9.7 & & \\
\hline CREYENTE MODERADO BAJO & 39 & 11.8 & & \\
\hline CREYENTE MODERADO ALTO & 97 & 29.3 & 331 & 2 \\
\hline BASTANTE CREYENTE RELIGIOSO & 115 & 34.7 & & \\
\hline MUY CREYENTE RELIGIOSO & 48 & 14.5 & & \\
\hline
\end{tabular}

(1)ESTAS PUNTUACIONES SON CENTIL 
Tabla 2.- RESULTADOS SEGÚN LOS SUBGRUPOS MUESTRALES PUNTUACIONES GLOBALES EN EL INVENTARIO DE CREENCIA RELIGIOSA

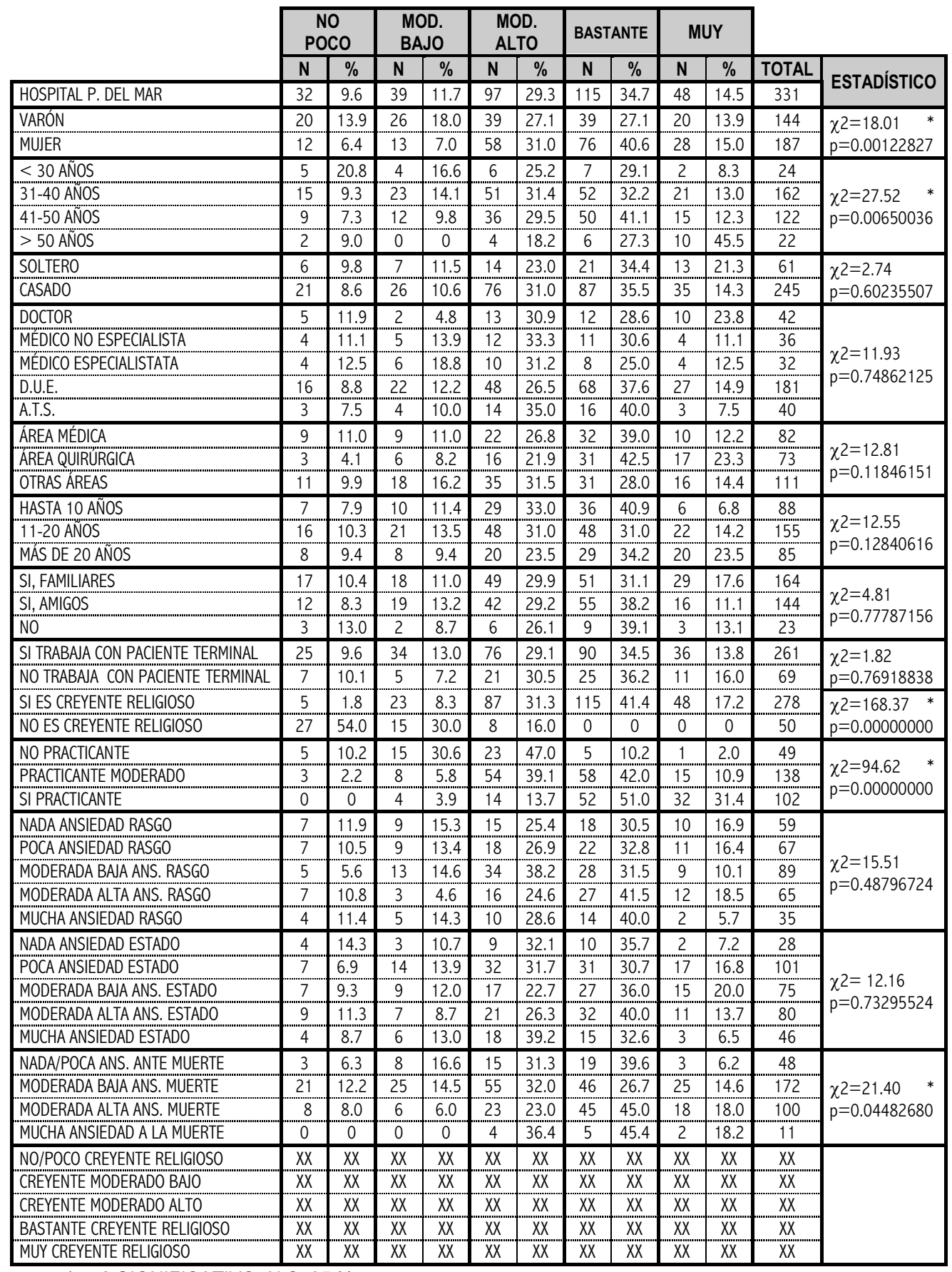

* $\chi^{2}$ SIGNIFICATIVO. N.C. $95 \%$. 
Tabla 3.- RESULTADOS SEGÚN LOS SUBGRUPOS MUESTRALES PUNTUACIONES GLOBALES EN LA ESCALA -DAS 1-

\begin{tabular}{|c|c|c|c|c|c|c|c|c|c|c|}
\hline & \multicolumn{2}{|c|}{$\begin{array}{l}\text { NADA } \\
\text { POCA }\end{array}$} & \multicolumn{2}{|c|}{$\begin{array}{l}\text { MODER. } \\
\text { BAJA }\end{array}$} & \multicolumn{2}{|c|}{$\begin{array}{l}\text { MODER. } \\
\text { ALTA }\end{array}$} & \multicolumn{2}{|c|}{ MUCHA } & & \multirow{3}{*}{ ESTADÍSTICO } \\
\hline & $\mathrm{N}$ & $\%$ & $\mathbf{N}$ & $\%$ & $\mathbf{N}$ & $\%$ & $\mathbf{N}$ & $\%$ & TOTAL & \\
\hline Hospital P. DEL MAR & 49 & 14.7 & 172 & 51.6 & 100 & 30.0 & 12 & 3.6 & 333 & \\
\hline VARÓN & 18 & 12.3 & 77 & 52.7 & 45 & 30.8 & 6 & 4.2 & 146 & \multirow{2}{*}{$\begin{array}{l}\chi 2=1.30 \\
p=0.72808219\end{array}$} \\
\hline MUJER & 31 & 16.6 & 95 & 50.8 & 55 & 29.4 & 6 & 3.2 & 187 & \\
\hline$<30$ AÑOS & 2 & 8.3 & 17 & 70.8 & 5 & 20.9 & 0 & 0 & 24 & \multirow{4}{*}{$\begin{array}{l}\chi^{2}=13.13 \\
p=0.15659776\end{array}$} \\
\hline 31-40 AÑOS & 26 & 16.0 & 87 & 53.7 & 45 & 27.7 & 4 & 2.6 & 162 & \\
\hline 41-50 ANOS & 17 & 13.8 & 57 & 46.3 & 44 & 35.8 & 5 & 4.1 & 123 & \\
\hline$>50$ ANOS & 3 & 13.0 & 11 & 47.8 & 6 & 26.1 & 3 & 13.1 & 23 & \\
\hline SOLTERO & 10 & 16.4 & 30 & 49.2 & 20 & 32.8 & 1 & 1.6 & 61 & \multirow{2}{*}{$\begin{array}{l}\chi 2=1.06 \\
p=0.78661443\end{array}$} \\
\hline CASADO & 36 & 14.6 & 126 & 51.2 & 74 & 30.1 & 10 & 4.1 & 246 & \\
\hline DOCTOR & 9 & 21.4 & 19 & 45.2 & 13 & 31.0 & 1 & 2.4 & 42 & \multirow{5}{*}{$\begin{array}{l}\chi^{2}=15.62 \\
p=0.20935617\end{array}$} \\
\hline MÉDICO NO ESPECIALISTA & 3 & 8.3 & 21 & 58.3 & 11 & 30.6 & 1 & 2.8 & 36 & \\
\hline MEDICO ESPECIALISTATA & 7 & 21.2 & 20 & 60.6 & 5 & 15.2 & 1 & 3.0 & 33 & \\
\hline D.U.E. & 21 & 11.5 & 99 & 54.4 & 56 & 30.8 & 6 & 3.3 & 182 & \\
\hline A.T.S. & 9 & 22.5 & 13 & 32.5 & 15 & 37.5 & 3 & 7.5 & 40 & \\
\hline ÁREA MÉDICA & 16 & 19.5 & 42 & 51.2 & 22 & 26.8 & 2 & 2.5 & 82 & \multirow{3}{*}{$\begin{array}{l}\chi 2=1.64 \\
p=0.94939554\end{array}$} \\
\hline ÁREA QUIRÜRGICA & 10 & 13.5 & 38 & 51.4 & 23 & 31.1 & 3 & 4.0 & 74 & \\
\hline OTRAS AREAS & 17 & 15.3 & 57 & 51.4 & 34 & 30.6 & 3 & 2.7 & 111 & \\
\hline HASTA 10 AÑOS & 16 & 18.2 & 47 & 53.4 & 23 & 26.1 & 2 & 2.3 & 88 & \multirow{3}{*}{$\begin{array}{l}\chi^{2}=10.03 \\
p=0.12333186\end{array}$} \\
\hline 11-20 ANOOS & 16 & 10.3 & 85 & 54.8 & 50 & 32.3 & 4 & 2.6 & 155 & \\
\hline MAS DE 20 ANOS & 17 & 19.5 & 37 & 42.5 & 27 & 31.0 & 6 & 7.0 & 87 & \\
\hline SI, FAMILIARES & 25 & 15.1 & 87 & 52.4 & 46 & 27.7 & 8 & 4.8 & 166 & \multirow{3}{*}{$\begin{array}{l}\chi^{2}=5.32 \\
p=0.50319147\end{array}$} \\
\hline SI, AMIGOS & 18 & 12.5 & 74 & 51.4 & 48 & 33.3 & 4 & 2.8 & 144 & \\
\hline NO & 6 & 26.1 & 11 & 47.8 & 6 & 26.1 & 0 & 0 & 23 & \\
\hline SI TRABAJA CON PACIENTE TERMINAL & 36 & 13.7 & 138 & 52.5 & 80 & 30.4 & 9 & 3.4 & 263 & \multirow{2}{*}{$\begin{array}{l}\chi 2=1.37 \\
p=0.71192460\end{array}$} \\
\hline NO TRABAJA CON PACIENTE TERMINAL & 13 & 18.8 & 34 & 49.3 & 19 & 27.5 & 3 & 4.4 & 69 & \\
\hline SI ES CREYENTE RELIGIOSO & 38 & 13.6 & 140 & 50.2 & 89 & 31.9 & 12 & 4.3 & 279 & \multirow{5}{*}{$\begin{array}{l}\chi^{2}=6.83 \\
p=0.07768301 \\
\chi_{2}=3.71 \\
p=0.71590975\end{array}$} \\
\hline NO ES CREYENTE RELIGIOSO & 11 & 21.6 & 30 & 58.8 & 10 & 19.6 & 0 & 0 & 51 & \\
\hline NO PRACTICANTE & 9 & 18.4 & 26 & 53.1 & 11 & 22.4 & 3 & 6.1 & 49 & \\
\hline PRACTICANTE MODERADO & 18 & 13.0 & 72 & 52.2 & 43 & 31.2 & 5 & 3.6 & 138 & \\
\hline SI PRACTICANTE & 13 & 12.6 & 49 & 47.6 & 37 & 35.9 & 4 & 3.9 & 103 & \\
\hline NADA ANSIEDAD RASGO & 11 & 18.7 & 32 & 54.2 & 14 & 23.7 & 2 & 3.4 & 59 & \multirow{5}{*}{$\begin{array}{l}\chi^{2}=10.72 \\
p=0.55331625\end{array}$} \\
\hline POCA ANSIEDAD RASGO & 10 & 14.9 & 40 & 59.7 & 15 & 22.4 & 2 & 3.0 & 67 & \\
\hline MODERADA BAJA ANS. RASGO & 12 & 13.5 & 47 & 52.8 & 26 & 29.2 & 4 & 4.5 & 89 & \\
\hline MODERADA ALTA ANS. RASGO & 7 & 10.6 & 31 & 47.0 & 27 & 40.9 & 1 & 1.5 & 66 & \\
\hline MUCHA ANSIEDAD RASGO & 4 & 11.4 & 15 & 42.9 & 14 & 40.0 & 2 & 5.7 & 35 & \\
\hline NADA ANSIEDAD ESTADO & 1 & 3.6 & 17 & 60.7 & 7 & 25.0 & 3 & 10.7 & 28 & \multirow{5}{*}{$\begin{array}{l}\chi^{2}=11.45 \\
p=0.49049331\end{array}$} \\
\hline POCA ANSIEDAD ESTADO & 17 & 16.7 & 56 & 54.9 & 26 & 25.5 & 3 & 2.9 & 102 & \\
\hline MODERADA BAJA ANS. ESTADO & 12 & 15.8 & 38 & 50.0 & 24 & 31.5 & 2 & 2.7 & 76 & \\
\hline MODERADA ALTA ANS. ESTADO & 14 & 17.5 & 38 & 47.5 & 25 & 31.2 & 3 & 3.8 & 80 & \\
\hline MUCHA ANSIEDAD ESTADO & 5 & 10.9 & 22 & 47.9 & 18 & 39.1 & 1 & 2.1 & 46 & \\
\hline NADA/POCA ANS. ANTE MUERTE & XX & $X X$ & $X X$ & $X X$ & $X X$ & $X X$ & $\overline{X X}$ & $X X$ & $\overline{X X}$ & \multirow{9}{*}{$\begin{array}{l}\chi 2=21.40 * \\
p=0.04482680\end{array}$} \\
\hline MODERADA BAJA ANS. MUERTE & $x X$ & $X X$ & $X X$ & $X X$ & $X X$ & $X X$ & $X X$ & $X X$ & $X X$ & \\
\hline MODERADA ALTA ANS. MUERTE & $X X$ & $X X$ & $X X$ & $X X$ & $\mathrm{XX}$ & $X X$ & $X X$ & $X X$ & $X X$ & \\
\hline MUCHA ANSIEDAD A LA MUERTE & $X X$ & $X X$ & XX & $X X$ & XX & $X X$ & $X X$ & $X X$ & $X X$ & \\
\hline NO/POCO CREYENTE RELIGIOSO & 3 & 9.3 & 21 & 65.7 & 8 & 25.0 & 0 & 0 & 32 & \\
\hline CREYENTE MODERADO BAIO & 8 & 20.5 & 25 & 64.1 & 6 & 15.4 & 0 & 0 & 39 & \\
\hline CREYENTE MODERADO ALTO & 15 & 15.4 & 55 & 56.7 & 23 & 23.8 & 4 & 4.1 & 97 & \\
\hline BASTANTE CREYENTE RELIGIOSO & 19 & 16.6 & 46 & 40.0 & 45 & 39.1 & 5 & 4.3 & 115 & \\
\hline MUY CREYENTE RELIGIOSO & 3 & 6.2 & 25 & 52.1 & 18 & 37.6 & 2 & 4.1 & 48 & \\
\hline
\end{tabular}

${ }^{*} \chi 2$ significativa. N.C. $95 \%$. 
Tabla 4.- RESULTADOS SEGÚN LOS SUBGRUPOS MUESTRALES PUNTUACIONES GLOBALES EN LA ESCALA - STAI "R" -

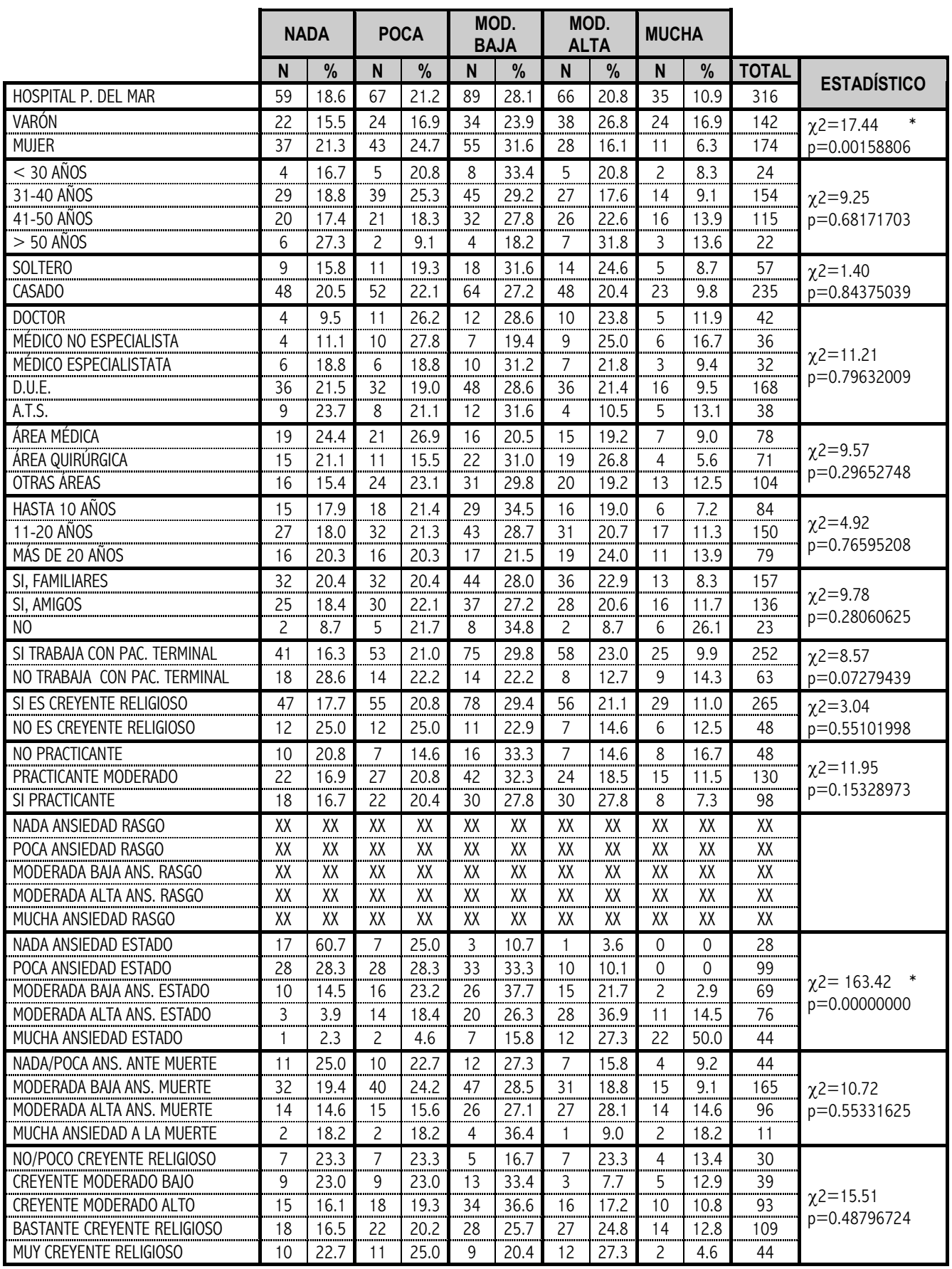

* $\chi 2$ SIGNIFICATIVO. N.C. $95 \%$. 
Tabla 5.- RESULTADOS SEGÚN LOS SUBGRUPOS MUESTRALES PUNTUACIONES GLOBALES EN LA ESCALA - STAI "E" -

\begin{tabular}{|c|c|c|c|c|c|c|c|c|c|c|c|c|}
\hline & \multicolumn{2}{|c|}{ NADA } & \multicolumn{2}{|c|}{ POCA } & \multicolumn{2}{|c|}{$\begin{array}{l}\text { MOD. } \\
\text { BAJA }\end{array}$} & \multicolumn{2}{|c|}{$\begin{array}{l}\text { MOD. } \\
\text { ALTA }\end{array}$} & \multicolumn{2}{|c|}{ MUCHA } & & \multirow{3}{*}{ ESTADÍSTICO } \\
\hline & $\mathrm{N}$ & $\%$ & $\mathbf{N}$ & $\%$ & $\mathrm{~N}$ & $\%$ & $\mathrm{~N}$ & $\%$ & $\mathrm{~N}$ & $\%$ & TOTAL & \\
\hline HOSPITAL P. DEL MAR & 28 & 8.4 & 102 & 30.7 & 76 & 22.8 & 80 & 24.0 & 46 & 13.8 & 332 & \\
\hline VARÓN & 10 & 6.9 & 40 & 27.6 & 38 & 26.2 & 31 & 21.4 & 26 & 17.9 & 145 & \multirow{2}{*}{$\begin{array}{l}\chi_{2}^{2}=6.66 \\
p=0.15518090\end{array}$} \\
\hline MUJER & 18 & 9.6 & 62 & 33.2 & 38 & 20.3 & 49 & 26.2 & 20 & 10.7 & 187 & \\
\hline$<30$ ANNOS & 2 & 8.3 & 9 & 37.5 & 3 & 12.5 & 6 & 25.0 & 4 & 16.7 & 24 & \multirow{4}{*}{$\begin{array}{l}\chi 2=3.37 \\
p=0.99227163\end{array}$} \\
\hline 31-40 ANOS & 12 & 7.4 & 47 & 29.0 & 41 & 25.3 & 41 & 25.3 & 21 & 13.0 & 162 & \\
\hline 41-50 ANOS & 12 & 9.9 & 38 & 31.1 & 27 & 22.1 & 27 & 22.1 & 18 & 14.8 & 122 & \\
\hline$>50$ ANOS & 2 & 8.7 & 8 & 34.8 & 5 & 21.7 & 5 & 21.7 & 3 & 13.1 & 23 & \\
\hline SOLTERO & 6 & 9.8 & 10 & 16.4 & 15 & 24.6 & 22 & 36.1 & 8 & 13.1 & 61 & \multirow{2}{*}{$\begin{array}{l}\chi^{2}=10.85 \\
p=0.02827856\end{array}$} \\
\hline CASADO & 17 & 6.9 & 88 & 35.9 & 57 & 23.3 & 52 & 21.2 & 31 & 12.7 & 245 & \\
\hline DOCTOR & 1 & 2.4 & 11 & 26.2 & 16 & 38.1 & 8 & 19.0 & 6 & 14.3 & 42 & \multirow{5}{*}{$\begin{array}{l}\chi^{2}=26.78 \\
p=0.04394873\end{array}$} \\
\hline MEDICO NO ESPECIALISTA & 1 & 2.8 & 10 & 27.8 & 5 & 13.9 & 11 & 30.5 & 9 & 25.0 & 36 & \\
\hline MEDICO ESPECIALISTATA & 2 & 6.3 & 10 & 31.3 & 9 & 28.0 & 6 & 18.8 & 5 & 15.6 & 32 & \\
\hline D.U.E. & 16 & 8.8 & 57 & 31.3 & 37 & 20.3 & 51 & 28.0 & 21 & 11.6 & 182 & \\
\hline A.T.S. & 8 & 20.0 & 14 & 35.0 & 9 & 22.5 & 4 & 10.0 & 5 & 12.5 & 40 & \\
\hline ÁREA MÉDICA & 13 & 15.9 & 21 & 25.6 & 22 & 26.8 & 15 & 18.3 & 11 & 13.4 & 82 & \multirow{3}{*}{$\begin{array}{l}\chi 2=15.40 \\
p=0.05189758\end{array}$} \\
\hline AREA QUIRURGICA & 6 & 8.2 & 24 & 32.9 & 18 & 24.7 & 20 & 27.4 & 5 & 6.8 & 73 & \\
\hline OTRAS AREAS & 4 & 3.6 & 38 & 34.3 & 21 & 18.9 & 32 & 28.8 & 16 & 14.4 & 111 & \\
\hline HASTA 10 AÑOS & 8 & 9.1 & 29 & 33.0 & 16 & 18.2 & 22 & 25.0 & 13 & 14.7 & 88 & \multirow{3}{*}{$\begin{array}{l}\chi 2=3.02 \\
p=0.93325676\end{array}$} \\
\hline 11-20 ANOS & 14 & 9.0 & 47 & 30.3 & 38 & 24.5 & 38 & 24.5 & 18 & 11.7 & 155 & \\
\hline MAS DE 20 ANOS & 6 & 7.0 & 24 & 27.9 & 22 & 25.6 & 20 & 23.3 & 14 & 16.2 & 86 & \\
\hline SI, FAMILIARES & 14 & 8.5 & 49 & 29.7 & 44 & 26.7 & 37 & 22.4 & 21 & 12.7 & 165 & \multirow{3}{*}{$\begin{array}{l}\chi^{2}=5.83 \\
p=0.66574990\end{array}$} \\
\hline SI, AMIGOS & 13 & 9.0 & 45 & 31.3 & 30 & 20.8 & 36 & 25.0 & 20 & 13.9 & 144 & \\
\hline NO & 1 & 4.3 & 8 & 34.8 & 2 & 8.7 & 7 & 30.4 & 5 & 21.8 & 23 & \\
\hline SI TRABAJA CON PAC. TERMINAL & 19 & 7.3 & 87 & 33.2 & 64 & 24.4 & 57 & 21.8 & 35 & 13.3 & 262 & \multirow{2}{*}{$\begin{array}{l}\chi 2=8.28 \\
p=0.08172873\end{array}$} \\
\hline NO TRABAJA CON PAC. TERMINAL & 9 & 13.0 & 15 & 21.7 & 12 & 17.4 & 22 & 31.9 & 11 & 16.0 & 69 & \\
\hline SI ES CREYENTE RELIGIOSO & 23 & 8.2 & 89 & 31.9 & 61 & 21.9 & 68 & 24.4 & 38 & 13.6 & 279 & \multirow{2}{*}{$\begin{array}{l}\chi 2=1.43 \\
p=0.83827702\end{array}$} \\
\hline NO ES CREYENTE RELIGIOSO & 5 & 10.0 & 13 & 26.0 & 14 & 28.0 & 11 & 22.0 & 7 & 14.0 & 50 & \\
\hline NO PRACTICANTE & 5 & 10.2 & 12 & 24.5 & 12 & 24.5 & 12 & 24.5 & 8 & 16.3 & 49 & \multirow{3}{*}{$\begin{array}{l}\chi^{2}=6.92 \\
p=0.54521739\end{array}$} \\
\hline PRACTICANTE MODERADO & 9 & 6.5 & 49 & 35.5 & 25 & 18.1 & 31 & 22.5 & 24 & 17.4 & 138 & \\
\hline SI PRACTICANTE & 9 & 8.7 & 31 & 30.1 & 27 & 26.2 & 26 & 25.2 & 10 & 9.8 & 103 & \\
\hline NADA ANSIEDAD RASGO & 17 & 28.8 & 28 & 47.4 & 10 & 17.0 & 3 & 5.0 & 1 & 1.7 & 59 & \multirow{5}{*}{$\begin{array}{l}\chi^{2}=163.42 \\
p=0.00000000\end{array}$} \\
\hline POCA ANSIEDAD RASGO & 7 & 10.4 & 28 & 41.8 & 16 & 23.9 & 14 & 20.9 & 2 & 3.0 & 67 & \\
\hline MODERADA BAJA ANS. RASGO & 3 & 3.4 & 33 & 37.0 & 26 & 29.2 & 20 & 22.5 & 7 & 7.9 & 89 & \\
\hline MODERADA ALTA ANS. RASGO & 1 & 1.5 & 10 & 15.2 & 15 & 22.7 & 28 & 42.4 & 12 & 18.2 & 66 & \\
\hline MUCHA ANSIEDAD RASGO & 0 & 0 & 0 & 0 & 2 & 5.7 & 11 & 31.4 & 22 & 62.9 & 35 & \\
\hline NADA ANSIEDAD ESTADO & $\mathrm{XX}$ & $x X$ & $x X$ & $\mathrm{XX}$ & $\mathrm{XX}$ & $X X$ & $x X$ & XX & $X X$ & $\mathrm{XX}$ & $\mathrm{XX}$ & \\
\hline POCA ANSIEDAD ESTADO & $\mathrm{XX}$ & XX & XX & $\mathrm{XX}$ & $\mathrm{XX}$ & $\mathrm{XX}$ & $X X$ & XX & $\mathrm{XX}$ & $\mathrm{XX}$ & $\mathrm{XX}$ & \\
\hline MODERADA BAJA ANS. ESTADO & $\mathrm{XX}$ & $\mathrm{XX}$ & XX & $\mathrm{XX}$ & $\mathrm{XX}$ & $\mathrm{XX}$ & $X X$ & XX & $\mathrm{XX}$ & $\mathrm{XX}$ & XX & \\
\hline MODERADA ALTA ANS. ESTADO & $\mathrm{XX}$ & $x X$ & XX & $\mathrm{XX}$ & $\mathrm{XX}$ & $\mathrm{XX}$ & $X X$ & XX & $\mathrm{XX}$ & $\mathrm{XX}$ & $\mathrm{XX}$ & \\
\hline MUCHA ANSIEDAD ESTADO & XX & $X X$ & XX & $\mathrm{XX}$ & $\mathrm{XX}$ & $X X$ & $X X$ & XX & $\mathrm{XX}$ & $\mathrm{XX}$ & $\mathrm{XX}$ & \\
\hline NADA/POCA ANS. ANTE MUERTE & 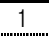 & 2.0 & 17 & 34.7 & 12 & 24.5 & 14 & 28.6 & 5 & 10.2 & 49 & \\
\hline MODERADA BAJA ANS. MUERTE & 17 & 9.9 & 56 & 32.8 & 38 & 22.2 & 38 & 22.2 & 22 & 12.9 & 171 & \\
\hline MODERADA ALTA ANS. MUERTE & 7 & 7.0 & 26 & 26.0 & 24 & 24.0 & 25 & 25.0 & 18 & 18.0 & 100 & $p=0.49049331$ \\
\hline MUCHA ANSIEDAD A LA MUERTE & 3 & 25.0 & 3 & 25.0 & 2 & 16.7 & 3 & 25.0 & 1 & 8.3 & 12 & \\
\hline NO/POCO CREYENTE RELLGIOSO & 4 & 12.9 & 7 & 22.6 & 7 & 22.6 & 9 & 29.0 & 4 & 12.9 & 31 & \\
\hline CREYENTE MODERADO BANO & 3 & 7.7 & 14 & 35.9 & 9 & 23.1 & 7 & 17.9 & 6 & 15.4 & 39 & \\
\hline CREYENTE MODERADO ALTO & 9 & 9.3 & 32 & 33.0 & 17 & 17.5 & 21 & 21.6 & 18 & 18.6 & 97 & 320 \\
\hline BASTANTE CREYENTE RELLGIOSO & 10 & 8.7 & 31 & 27.0 & 27 & 23.5 & 32 & 27.8 & 15 & 13.0 & 115 & \\
\hline MUY CREYENTE RELIGIOSO & 2 & 4.1 & 17 & 35.4 & 15 & 31.3 & 11 & 22.9 & 3 & 6.3 & 48 & \\
\hline
\end{tabular}

* $\chi 2$ SIGNIFICATIVO. N.C. $95 \%$. 


\section{BIBLIOGRAFÍA:}

I. Pastor G: Ideologías. Ed. Herder. Barcelona, 1986.

2. Nee W: El hombre espiritual: Espíritu, alma y cuerpo. Ed. Clie Terrasa. Barcelona, 1988.

3. Rodríguez A y Seoane J: Creencias, Actitudes y Valores. V. 7 Tratado de Psicología General de J. Mayor y J. Pinillos. Ed. Alhambra Universidad. Madrid, 1989.

4. Bedoya J: Actitudes básicas y religión. Tesis Doctoral. Universidad de Málaga, 1995.

5. Blanco A y Giner ]: Asistencia psicológica al moribundo al final de su vida. En A. Selva, Bases Psicológicas de los estados de salud y enfermedad. INO Reproducciones S.A. Madrid, 1994.

6. Gray V: Algunas necesidades psicológicas. En P. Vilagrasa y M. Peya, Paciente Terminal y muerte. Ed. Doyma. Barcelona, 1987.

7. Urraca S: Actitudes ante la muerte (preocupación, ansiedad, temor) y religiosidad. Tesis doctoral. Universidad Complutense. Madrid, 1982.

8. Lonetto R y Templer D: Ansiedad ante la muerte. Edic. especial para Zembeleti España, S.A. Barcelona, 1988.

9. Aries A: El hombre ante la muerte. Ed. Taurus. Madrid, 1987.

10. Gala FJ, Lupiani M y Díaz M: Sobre las concepciones de la muerte. Rev. Rol, 1991;159: 63-66.

II. Hinton J: Experiencias sobre el morir. Ed. Seix Barral. Barcelona, 1996.

12. Cortes H, Wickham P y Martínez P: La muerte, dolor, sufrimiento y eutanasia. Una visión cristiana. Cuadernos de Ética Pastoral. Alianza Evangélica Española. Barcelona, 1997.

13. Pardo de Velez G y Cedeño M: Investigación en Salud. Ed. McGrawHill. Santafé de Bogotá, 1997.

14. Bazerque R y Tesler C: Métodos y técnicas de la investigación clínica. Ed.Toray-Masson. París 1982.

15. Carrasco J: El método estadístico en la investigación médica. Ed. Ciencia 3. Madrid 1988.

16. Aycaques S: Muestreo para la investigación en ciencias de la salud. Ed. Díaz de Santos. Madrid 1994.

17. Fisher L y Yates S: Statistical tables. Edit. Conginan. Nueva York 1974.

18. Spielberger CD, Gorsuch RL y Lushene RE: Cuestionario de ansiedad estado-rasgo (STAI). Edit. TEA. Madrid 1986.

19. Templer DI: The construction and validation of a death anxiety scale. Journal of General Psychology, 1970; 82: 165-177.

20. Raja R: Influencia de las Creencias Religiosas en las actitudes del P.S. ante la muerte. Tesis doctoral. Universidad de Cádiz. 200I.

2I. C.D.C: Epidemiology program office. (EPI-INFO 3 GA 30333). Atlanta, 1989.

22. C.D.C. y 0.M.S. (Dean A y Burton A): Public Domain Sofware for epidemiology and disease surveillance (EPI-INFO 6.04). Publicaciones del C.D.C. Atlanta, 1996.

23. Gala FJ, Lupiani M, Díaz M, et al: Actitudes hacia el SIDA entre profesionales sanitarios. Rev. Scientia, 1997; V III-I:51-60.

24. Amon y Yela M: Dimensiones de la religiosidad. Rev. de Psicología General y Aplicada, 1968; 95: 989-993.

25. Hemenway JE: ¿Dónde está Dios?. En 0.J.Z. Shaley, El niño y la muerte (pp 217-225). Ed. Alhambra. Madrid 1983.

26. Revista Muy Interesante. Sep, 2001.

27. Amon y Yela M: Op. Cit. 1968.
28. Vega J y Bueno B: Desarrollo adulto y envejecimiento. Ed. Síntesis. Madrid, 1995.

29. Garzón A y Garcés ]: Hacia una conceptualización del valor. En J. Mayor y J.L. Pinillos (eds), Creencias, Actitudes y Valores. Tratado de Psicología General n0 7. Ed. Alhambra. Madrid.1989, pp 365-407.

30. Papalia D y Wendkos S: Psicología del desarrollo (5 $5^{a}$ Edición) Ed. MacGraw-Hill Interamericana. México 1999.

31. Pacheco R: Estudio de actitudes ante la muerte y los enfermos terminales en médicos y ATS con ejercicio profesional en la ciudad de Murcia. Tesis Doctoral. Universidad de Murcia, 1993.

32. Blanco A: El médico ante la muerte de su paciente. Ed. Monardes. Sevilla, 1992.

33. Ramos C y García I: Miedo y ansiedad ante la muerte. En ]. Buendía, Psicología Clínica y Salud : Desarrollos actuales. Universidad de Murcia. 199I, pp I3I-I60.

34. Kübler-Ross E: On death and dying. Ed. Macmillan. New York 1969.

35. Weisman AD: On dying and denying: A psychiatric study of terminality. Behavioral Publications. New Yorks 1972.

36. Ramos C y García I: Op. Cit. 1991.

37. Templer D, Barthlow V, Halcomb P et al: The death anxiety of convicted persons. Conective and Social Psychiatry, 1979; 25:18-25.

38. Moraleda M: Psicología del desarrollo. Ed. Boixareu Universitaria. Barcelona 1989.

39. Serra E, Gonzalez A, y Oller A: Desarrollo adulto. Grupo Editor Universitario. Barcelona, 1979

40. Feifel H y Branscomb AB: Who's afraid of death?. Journal of Abnormal Psychology, 1973; 81: 282-288.

4I. Leming MR: The relationship between religiosity and the fear of death. Dissertation Abstracts International, 1976; 36: II.

42. lanmarino NK: Relationship between death anxiety and demographic variables. Psychological Reports, 1976; 37: 362.

43. Macdonald GW: Sex, religion, and risk-taking behavior as correlates of death anxiety. Omega, 1976; 7: 35-44.

44. Templer DI y Dotson E: Religious correlates of death anxiety. Psychological Reports, 1970; 26: 895-897.

45. Ramos C y García I: Op. Cit. 1991.

46. Gala FJ y Guillén C: El estrés y la ansiedad: males de nuestra época. Servicio de Publicaciones de la Universidad de Cádiz. 2000.

47. Hernández-Monsalve L y Gervas ]: Estrés en los Profesionales Sanitarios. En J. Gestal (Ed.), Riesgos del Trabajo del Personal Sanitario .Ed. MacGraw-Hill. Madrid, 1993, pp 447-457

48. Spielberger CD; Gorsuch RL y Lushene RE: 0p. Cit. 1986.

49. Blechman E y Brownell K: Medicina conductual de la mujer. Ed. Martínez Roca. Barcelona, 1992.

50. Maccoby E: Desarrollo de las diferencias sexuales. Ed. Marova. Madrid, 1982.

5I. Pacheco R: Op. Cit. 1993.

52. Gala FJ, Barba A, Pérez A et al: Ansiedad producida por estímulos sanitarios desagradables. Rev. Rol, 1990; 143-144: 60-63.

53. Alonso F: Salud mental y Morbilidad psiquiátrica en el personal sanitario. En J. Gestal (Ed), Riesgo del trabajo del personal sanitario. Ed. McGraw-Hill. Interamericana. Madrid, 1993, pp 4I4-423. 